\title{
The association between depression and metabolic syndrome and its components: a bidirectional two-sample Mendelian randomization study
}

\author{
Min Zhang ${ }^{1}$, Jing Chen ${ }^{2}$, Zhiqun Yin $^{3}$, Lanbing Wang ${ }^{4}$ and Lihua Peng $\mathbb{D}^{2 凶}$ \\ (c) The Author(s) 2021
}

\begin{abstract}
Observational studies suggested a bidirectional correlation between depression and metabolic syndrome (MetS) and its components. However, the causal associations between them remained unclear. We aimed to investigate whether genetically predicted depression is related to the risk of MetS and its components, and vice versa. We performed a bidirectional two-sample Mendelian randomization (MR) study using summary-level data from the most comprehensive genome-wide association studies (GWAS) of depression $(n=2,113,907)$, MetS $(n=291,107)$, waist circumference $(n=462,166)$, hypertension $(n=463,010)$ fasting blood glucose (FBG, $n=281,416$ ), triglycerides ( $n=441,016)$, high-density lipoprotein cholesterol (HDL-C, $n=403,943)$. The random-effects inverse-variance weighted (IVW) method was applied as the primary method. The results identified that genetically predicted depression was significantly positive associated with risk of MetS (OR: 1.224, 95\% Cl: $1.091-1.374, p=5.58 \times 10^{-4}$ ), waist circumference (OR: $1.083,95 \% \mathrm{Cl}: 1.027-1.143, p=0.003)$, hypertension (OR: $1.028,95 \% \mathrm{Cl}: 1.016-1.039, p=1.34 \times 10^{-6}$ ) and triglycerides (OR: $1.111,95 \% \mathrm{Cl}: 1.060-1.163, p=9.35 \times 10^{-6}$ ) while negative associated with HDL-C (OR: 0.932, 95\% Cl: $0.885-0.981$, $p=0.007$ ) but not FBG (OR: $1.010,95 \% \mathrm{Cl}: 0.986-1.034, p=1.34)$. No causal relationships were identified for MetS and its components on depression risk. The present MR analysis strength the evidence that depression is a risk factor for MetS and its components (waist circumference, hypertension, FBG, triglycerides, and HDL-C). Early diagnosis and prevention of depression are crucial in the management of MetS and its components.
\end{abstract}

Translational Psychiatry (2021)11:633; https://doi.org/10.1038/s41398-021-01759-z

\section{INTRODUCTION}

Depression is a common mental health illness. The lifetime prevalence of depression varies across countries with a midpoint of about $10.0 \%$ and is generally higher in higher-income than lower-income countries [1-3]. Globally, depression is estimated to be one of the leading causes of "years lost" to a disability, resulting in a loss of individual productivity, placing a severe economic burden [4-6]. In its most severe cases, depression can lead to suicide [7-9], contributing to the increased risk of mortality [10]. With the increasing incidence [11], depression is one of the most challenging public health issues today.

Another big health threat is metabolic syndrome (MetS), which is defined as a clustering of abdominal obesity, hyperglycemia, elevated blood pressure, and dyslipidemia [12]. The prevalence of MetS varies in different ethnicity due to the criteria used for MetS definitions and has steadily risen worldwide [13, 14]. It was estimated that MetS has affected over a billion people globally [13]. MetS and its components are associated with various diseases and overall mortality, making it a severe health problem and economic burden $[15,16]$.

As both depression and MetS are risk factors for cardiovascular disease $[15,17]$, their relationship has been attracted extensive attention in recent years $[18,19]$. Epidemiological data have consistently indicated a co-occurrence of depression with MetS and its components [20-22]. Several meta-analyses and reviews also suggested a bidirectional relationship between depression and MetS [23-26]. It has been shown that depression increased MetS risk by $34 \%$ in cross-sectional studies and $52 \%$ in cohort studies. Conversely, patients with MetS had a 1.27-fold (crosssectional studies) to 1.49 times (cohort studies) higher risk of depression than controls [23]. However, the bidirectional correlations between depression and MetS and its components identified in observational studies might be susceptible to confounding factors, small sample size, limited follow-up time, and reverse causation, which might mislead the conclusion [27]. Thus, the potential causality of depression in determining the risk of MetS and its components remains elusive and vice versa.

Mendelian randomization (MR) is a more robust method of causal inferences, which could overcome the limitations of observational studies $[28,29]$. MR uses genetic variations that are robustly associated with exposure as an instrument, which might effectively avoid the influence of confoundings and reverse causes [28, 30-32]. Genome-wide association studies (GWAS) have identified thousands of variants related to complex exposures,

\footnotetext{
${ }^{1}$ School of Public Health and Management, Chongqing Medical University, Chongqing 400016, China. ${ }^{2}$ Department of Anesthesia and Pain Medicine, The First Affiliated Hospital of Chongqing Medical University, Chongqing 400016, China. ${ }^{3}$ Department of Psychiatry and Psychology, No.964 Hospital of People's Liberation Army, Changchun City 130026 Jilin Province, China. ${ }^{4}$ Division of medical affairs, The First Affiliated Hospital of Army Military Medical University, Chongqing 400038, China. ${ }^{凶}$ email: plhcqmu@163.com
} 
A

\begin{tabular}{llll}
\hline MR analysis & \multicolumn{2}{c}{ The reverse MR analysis } \\
Exposure & Outcome & Exposure & Outcome \\
\hline Depression & Metabolic syndrome & Metabolic syndrome & Depression \\
Depression & Waist circumference & Waist circumference & Depression \\
Depression & Hypertension & Hypertension & Depression \\
Depression & Fasting blood glucose & Fasting blood glucose & Depression \\
Depression & Triglycerides & Triglycerides & Depression \\
Depression & HDL-C & HDL-C & Depression \\
\hline
\end{tabular}

B

Assumptions:

(1) genetic instruments are significantly associated with exposure of interest;

(2) genetic instruments are not related to any confounding factors of the exposureoutcome association;

(3) genetic instruments affect the outcome only via the exposure.



Fig. 1 Overview of the study design in this bidirectional MR study. A We performed a total of 12 MR analyses to investigate the bidirectional association between depression and MetS and its components. B MR analysis depends on three key assumptions. C Sketch of the study design. MetS metabolic syndrome, FBG fasting blood glucose, TG triglycerides, WC waist circumference, HDL-C high-density lipoprotein cholesterol.

which has pushed the widespread use of MR to a new climax $[33,34]$. In this study, we utilized a bidirectional two-sample MR method to examine bidirectional causality between depression and MetS and its components.

\section{MATERIALS AND METHODS Study design overview}

A brief description of this bidirectional MR design is shown in Fig. 1. The components of MetS were charactered with five elements according to the National Cholesterol Education Program Adult Treatment Panel III (NCEP/ ATP III) criteria [35]. We tested the causal relationship of depression on MetS and its components. We adopted the summary-level statistics from the most comprehensive meta-analyses of GWASs for MetS, hypertension, waist circumference, fasting blood glucose (FBG), serum triglycerides (TG), and serum high-density lipoprotein cholesterol (HDL-C). In the reversedirection MR analysis, we assessed the association between genetically predicted MetS and its components and depression risk. Summary-level data from the most extensive meta-analyses of GWASs for depression was also extracted. Thus, we performed a total of $12 \mathrm{MR}$ analyses to investigate the bidirectional association between depression and metabolic syndrome and its components. MR depends on three key assumptions (Fig. 1): (1) genetic instruments are significantly associated with exposure of interest; (2) genetic instruments are not related to any confounding factors of the exposure-outcome association; (3) genetic instruments affect the outcome only via the exposure [36]. Details of the data sources used in this study are summarized in Supplementary Table 1. To minimize racial mismatches, our analyses are restricted to most of the participants of European descent.

\section{Selection of genetic instruments for MR analyses}

For each exposure factor, the SNPs were filtered according to the three main assumptions of MR. Firstly, we included SNPs at a threshold of genome-wide significance $\left(p<5 \times 10^{-8}\right)$. Then, we retained variants with the lowest $p$ value as independent instruments based on linkage disequilibrium (LD) as measured by $r^{2}$ (when $r^{2}>0.1$ in the European 1000 Genome reference panel). Finally, to quantify the strength of instrumental variables, we calculated $F$-statistics, and a threshold of the $F$ statistics $>10$ was typically recommended for MR analyses.

\section{Data sources and SNP selection for depression}

We used the hitherto largest published GWAS for depression, which included participants from 23andMe, Psychiatric Genomics Consortium (PGC), and UK Biobank with a total of $2,113,907$ subjects $(660,418$ cases and $1,453,489$ controls) [37]. Totally, 97 independent genetic SNPs were identified with genome-wide significant levels $\left(p<5 \times 10^{-8}\right)$ and were selected as genetic instruments for depression. The reverse-direction MR analyses used the summary-level data from PGC and UK Biobank with 500,199 individuals, which were publicly available (Supplementary Table 1).

\section{Data sources and SNP selection for MetS}

Summary-level data for MetS were taken from the most comprehensive GWAS in UK Biobank [38], consisting of 291,107 individuals (59,677 cases and 231,430 controls) with nonmissing data on genotypes, outcomes, and covariates. For reverse MR analyses, 79 independent loci associated with MetS were identified in this GWAS with a genome-wide threshold of significance $\left(p<5 \times 10^{-8}\right)$, and we selected the satisfying variants for the construction of the instrumental variables (Supplementary Table 1).

Data sources and SNP selection for the components of MetS For waist circumference, we extracted the GWAS summary data from the Medical Research Council Integrative Epidemiology Unit (MRC-IEU) UK Biobank GWAS Pipeline, which included 462,166 subjects with European ancestry [39]. In the reverse-direction MR analyses, we selected 31 variants with a $p$ value less than $5 \times 10^{-8}$ recognized from a GWAS [40], which included up to 224,459 individuals from the Genetic Investigation of ANthropometric Traits (GIANT) Consortium for the construction of genetic instruments for waist circumference (Supplementary Table 1).

As to hypertension, the summary statistics were also available from the MRC-IEU UK Biobank pipeline, which included 463,010 subjects [39]. Twelve variants associated with hypertension at a genome-wide significance were identified from a GWAS of 29 studies [41] with 203,006 
participants (GWAS: 69,395, and follow-up: 133,611) of European descent, and we selected those SNPs as the genetic instruments for hypertension (Supplementary Table 1).

For FBG, we obtained the summary-level data from the most comprehensive GWAS in the meta-analyses of glucose and insulin-related traits Consortium (MAGIC), which included 281,416 individuals with over $70 \%$ were European ancestry and were publicly available data [42]. A total of 134 SNPs were identified to be significantly associated with FBG $(p<5 \times$ $10^{-8}$ ) and were selected as instruments for FBG (Supplementary Table 1).

For TG and HDL-C, the summary-level statistics were extracted from the UK Biobank with above 400,000 participants [43]. For the reverse-direction MR analyses, the variants were extracted from a most representative GWAS of 188,577 subjects from the Global Lipids Genetics Consortium (GLGC) [44]. This GWAS identified 28 and 60 SNPs significantly associated with TG and HDL-C $\left(p<5 \times 10^{-8}\right)$, respectively, and were used as instrumental variables (Supplementary Table 1).

\section{Statistical analyses}

In this study, $R^{2}$ was calculated to represent the proportion of variance in an exposure factor explained by the instrumental variables. F-statistic was calculated to represent the strength of the association between the instruments and risk of exposure of interest [45]. For binary exposures, the casual estimate was presented as an odds ratio (OR) and 95\% confidence interval $(\mathrm{Cl})$ per log-odds increment in genetically determined risk of the exposures. As to continuous exposures, the causal estimate was presented as an OR with a $95 \% \mathrm{Cl}$ per standard deviation (SD) increment in an exposure. MR analyses utilized the random-effects inverse-variance weighted (IVW) method as the primary method to estimate the potential bidirectional causal associations between depression and MetS and its components as it provides a robust causal estimate in the absence of directional pleiotropy. Furthermore, we used weighted median, simple mode, weight mode, and MR-Egger methods for alternative analyses. Then, we performed tests for directional horizontal pleiotropy by MR-Egger intercept. We also tested the heterogeneity for MR-Egger regression and IVW method via Cochran's $Q$ statistics and funnel plots $[46,47]$. In addition, sensitivity analysis by applying leave-one-out analysis was conducted. Post hoc power calculation for MR was based on online web tools (https://sb452. shinyapps.io/power/) [48]. All statistical analyses were performed using the TwoSampleMR packäges in R (version 3.6.3, www.r-project.org/) or Stata 16 (Stata, College Station, TX). All $p$ values are two-tailed. We adopted Bonferroni-corrected $p$ value $<0.004(0.05 / 12=0.004)$ to determine statistical significance in the MR analysis, and a $p$ value less than 0.10 was considered significant in the MR-Egger test and heterogeneity test.

\section{RESULTS}

The causal effect of depression on MetS and its components Among the 97 depression-associated variants, two SNPs were not available in the summary-level datasets of wais circumference, hypertension, and triglycerides, and three SNPs were unavailable for HDL-C datasets. In addition, due to ambiguous palindrome, we excluded one variant for MetS and FBG, six variants for waist circumference and hypertension, five SNPs for triglycerides, and four SNPs for HDL-C. Thus, we finally included 96, 89, 89, 96, 90, and 90 variants as genetic instruments for MetS, waist circumference, hypertension, FBG, triglycerides, and HDL-C in the MR analyses, respectively. The $R^{2}$ and F-statistics indicated that all genetic instruments were suitable for MR analysis, and most of the statistical power to test an OR of 1.20 was higher than $80.0 \%$ (Table 1).

The results of the MR analyses were shown in Table 2, the scatter plots and forest plots were presented in Fig. 2 and Supplementary Fig. 1, respectively. Genetically predicted depression was significantly positively associated with MetS. The OR with $95 \% \mathrm{Cl}$ of per log_odds increment in depression liability was 1.224 (95\% Cl: $\left.1.091-1.374 ; p=5.58 \times 10^{-4}\right)$ in the IVW model, which was consistent with the result of the weight median model. MREgger regression analysis showed no indication of potential nonhorizontal pleiotropy (egger_intercept $=0.002, p=0.747$ ). The Cochran's $Q$ value suggested an obvious heterogeneity $(Q=$ $271.41, p<0.001$ ) obtained from individual variants, but the funnel plot (Supplementary Fig. 2) showed an invisible asymmetry of the MR analyses, indicating no evidence of heterogeneity. Furthermore, the leave-one-out analysis suggested that the observed association was not significantly changed after removing any single variant (Supplementary Fig. 3).

As to its components, genetic liability to depression was positively related to waist circumference, hypertension, and triglycerides while negatively associated with HDL-C. The ORs with $95 \%$ Cls per log_odds increment in genetically predicted depression were 1.083 (95\% Cl: $1.027-1.143 ; p=0.003)$ for waist circumference, 1.028 (95\% Cl: $\left.1.016-1.039 ; p=1.34 \times 10^{-6}\right)$ for hypertension, $1.111\left(95 \% \mathrm{Cl}\right.$ : $\left.1.060-1.163 ; p=9.35 \times 10^{-6}\right)$ for triglycerides, and 0.932 (95\% Cl: 0.885-0.981, $p=0.007$ ) for HDL-C in the IVW models (Table 2). No significantly association was identified for $\mathrm{FBG}(\mathrm{OR}=1.010 ; 95 \% \mathrm{Cl}$ : $0.986-1.034 ; p=0.423)$. Those associations were mostly consistent across other models. MR-Egger regression analyses indicated that there was no potential horizontal pleiotropy (all $p$ values $>0.10$ ) (Table 2). As to heterogeneity, Cochran's $Q$ test indicated obviously heterogeneities (all $p$ values of Cochran's $\mathrm{Q}<0.001$ ), however, most of the funnel plots suggested no evidence of heterogeneity (Supplementary Fig. 2). In addition, the leave-one-out analysis showed that the results were not significantly changed after

Table 1. The $R^{2}$ and F-statistics for the genetic instruments and the power for MR.

\begin{tabular}{|c|c|c|c|c|c|}
\hline Exposure & Outcome & No. SNP & $R^{2}$ & F-statistic & Power \\
\hline Depression & Metabolic syndrome & 96 & $0.37 \%$ & 77.71 & $99.6 \%$ \\
\hline Depression & Waist circumference & 89 & $0.33 \%$ & 78.75 & $90.9 \%$ \\
\hline Depression & Hypertension & 89 & $0.32 \%$ & 77.19 & $90.4 \%$ \\
\hline Depression & Fasting blood glucose & 96 & $0.32 \%$ & 77.99 & $73.2 \%$ \\
\hline Depression & Triglycerides & 90 & $0.33 \%$ & 78.47 & $89.8 \%$ \\
\hline Depression & HDL-C & 90 & $0.33 \%$ & 77.00 & $86.5 \%$ \\
\hline Waist circumference & Depression & 30 & $0.76 \%$ & 55.57 & $100.0 \%$ \\
\hline Hypertension & Depression & 10 & $0.22 \%$ & 37.21 & $100.0 \%$ \\
\hline Fasting blood glucose & Depression & 118 & $13.56 \%$ & 230.46 & $100.0 \%$ \\
\hline Triglycerides & Depression & 26 & $2.84 \%$ & 196.14 & $100.0 \%$ \\
\hline
\end{tabular}

$H D L-C$ high-density lipoprotein cholesterol. 




$m$
0
0

客

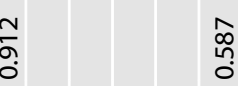

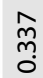

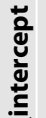

离

ঃ

ঃ

\&

sọ

ô

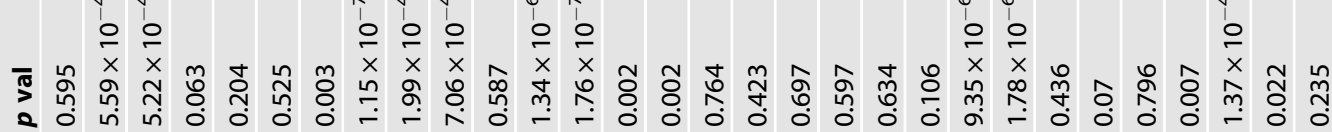

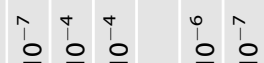

i

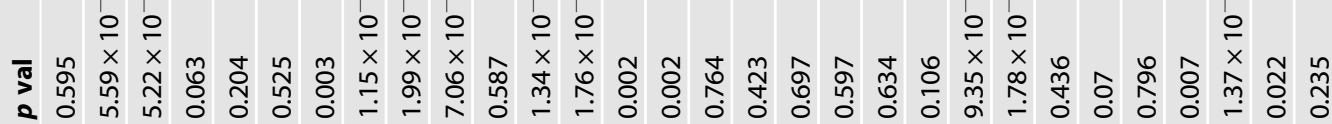

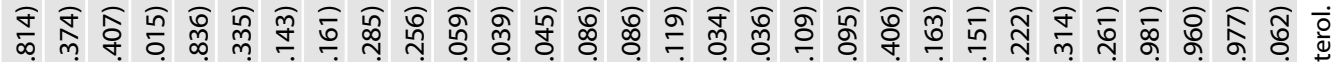
= I T T 官全 ĥे

茂

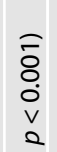



$\overline{\bar{o}}$

ò

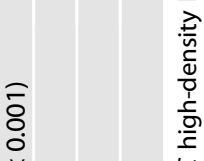

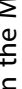

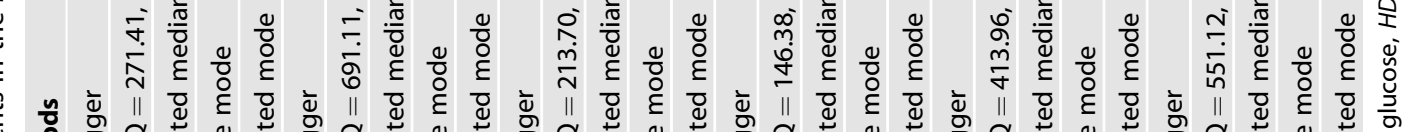

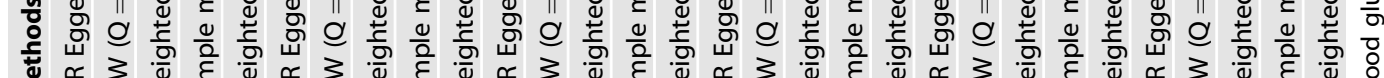

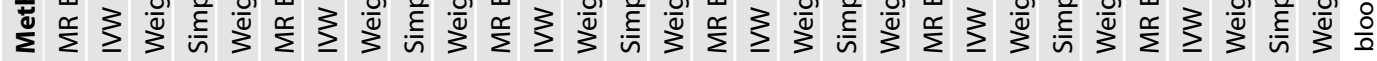

产

2

$\infty$

ळ

ஜ

8

8

党

旁

ธ

흠

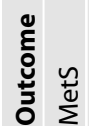

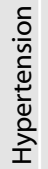

ญ્



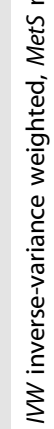



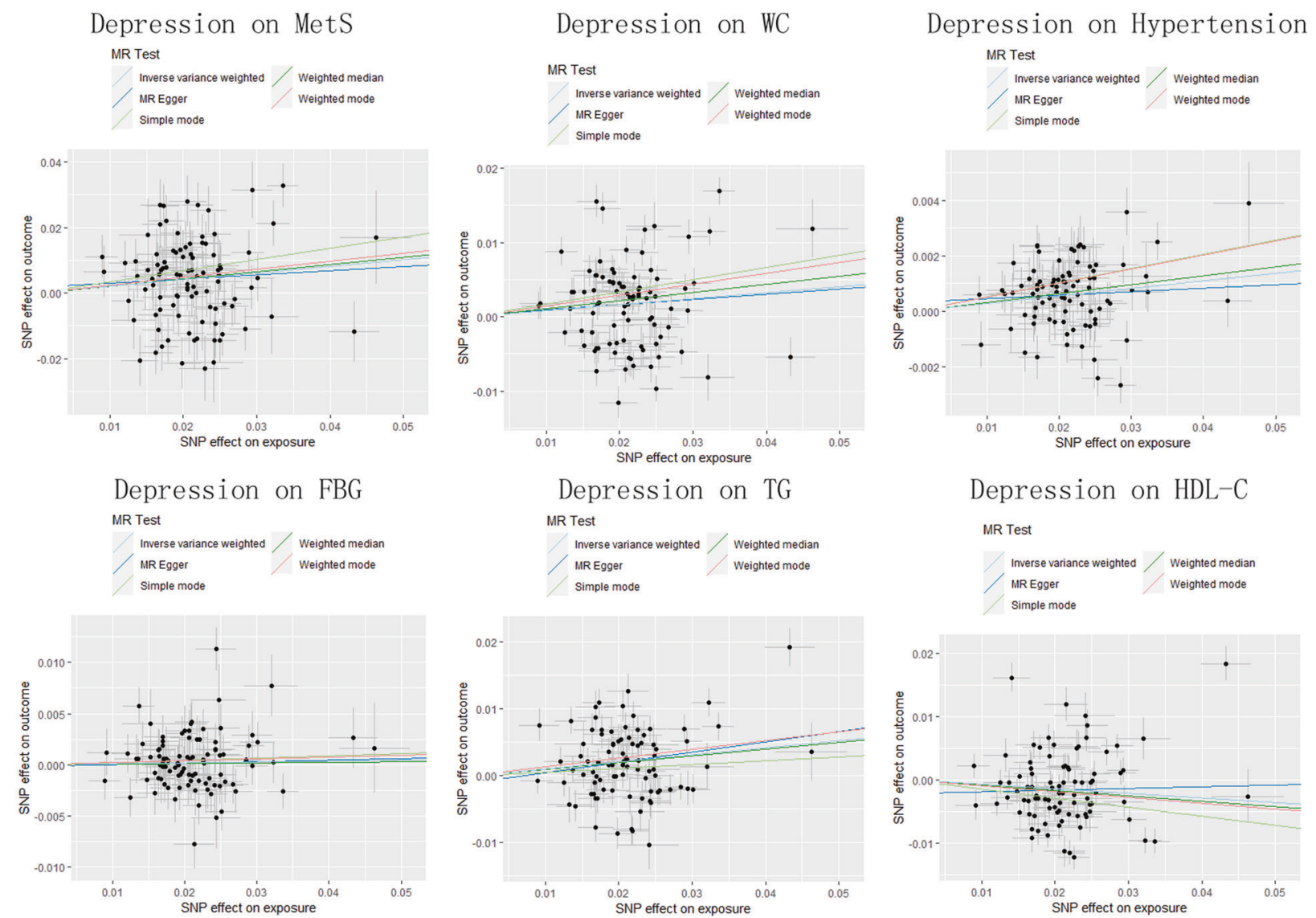

Depression on HDL-C

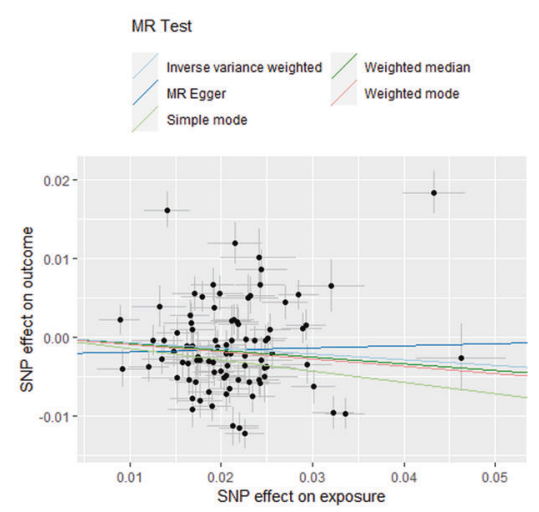

Fig. 2 The scatter plots of the association between genetically predicted depression on MetS and its components. MetS metabolic syndrome, FBG fasting blood glucose, TG triglycerides, WC waist circumference, HDL-C high-density lipoprotein cholesterol.

omitting any single SNP, suggesting the stability of the observed associations (Supplementary Fig. 3).

The causal effect of MetS and its components on depression In the reverse MR analysis, after excluding the unavailable SNPs in the summary-level dataset of depression and the palindromic SNPs, we utilized 73 variants for MetS, 30 variants for waist circumference, ten variants for hypertension, 118 variants for FBG, 26 variants for triglycerides, and 60 variants for $\mathrm{HDL}-\mathrm{C}$ as genetic instruments, respectively. We had high statistical power $(>80 \%$ power to estimate an OR of 1.20) to assess associations of MetS and its components with depression (Table 1).

As shown in Table 3, the scatter plots (Supplementary Fig. 4), and forest plots (Supplementary Fig. 5), the MR results showed neither MetS nor its five elements were causally related to depression, with ORs close to 1. Egger's test showed no potential horizontal pleiotropy exists but for the relationship between waist circumference and FBG and risk of depression. Cochran's $Q$ test and funnel plots (Supplementary Fig. 6) indicated obviously heterogeneities except for the association between hypertension and risk of depression. The leave-one-out analysis also revealed the stability of the results (Supplementary Fig. 7).

\section{DISCUSSION}

In this bidirectional two-sample MR study, we found that genetically predicted depression was significantly positively associated with risk of MetS, waist circumference, hypertension, and triglycerides while negatively associated with HDL-C. The reverse MR analyses observed no evidence that liability to MetS and its components was associated with depression.
Epidemiological data have been consistently proposed that depression was positively correlated with MetS risk [20, 49, 50]. Several meta-analyses of cohort studies suggested that depression was an independent risk factor for MetS, which agrees with our results $[24,25]$. As to the reverse direction, observational studies on the effect of MetS on depression risk were inconclusive. Some cohort studies found that MetS could not independently predict depression risk [51, 52], however, some publications proposed a significant positive association $[53,54]$. Our MR analysis showed no evidence supporting a determinate causal effect of MetS on depression, suggesting that the observed association is likely due to confoundings, such as physical activity [55, 56] and diet [57]. In addition, Koponen et al. found that MetS was a risk factor for depression only in females [58]. A prospective cohort study in the French population showed MetS associated with new-onset depressive symptoms in the younger-aged group but not in older-aged subjects [53]. Those data suggested that depression risk in the MetS population might differ depending on the definition of MetS and the participants' characteristics such as age and sex.

As for the components of MetS, a meta-analysis suggested depression was positively associated with central obesity [59]. In addition, subjects with depression have a significant increase in triglycerides levels and a decrease in HDL-C levels [21, 60]. A crosssectional study in Iran also showed that depression was associated with elevated blood pressure in the elderly, especially men [61]. Consistent with these evidence, our MR analyses proposed that genetically predicted depression was correlated with high waist circumference (an indicator of abdominal obesity) and triglycerides, lower HDL-C, and hypertension. However, we did not identify a causal relationship between depression and high FBG, although some observational studies pointed to an epidemiological 

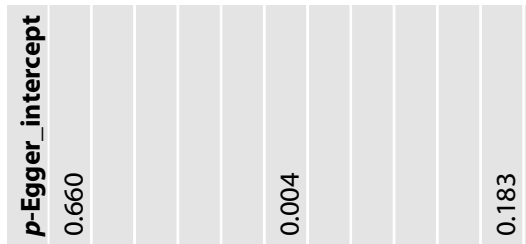

$\stackrel{0}{0}$

京

m.
0

๖.

웅

ọ̊

웅

훙

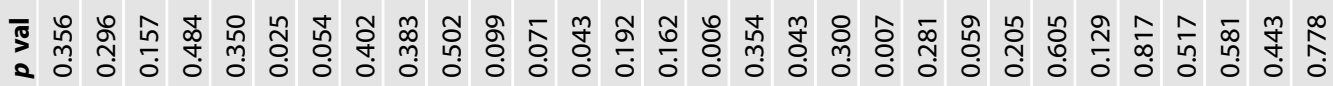

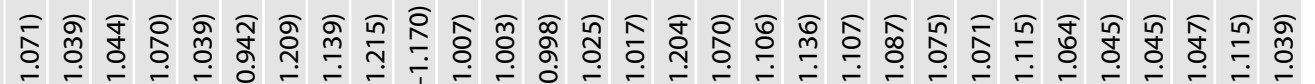

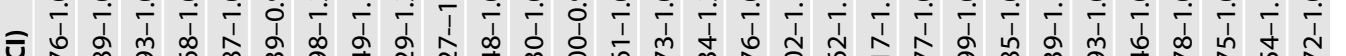

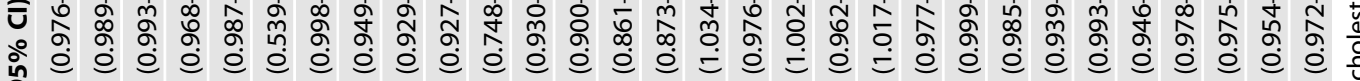

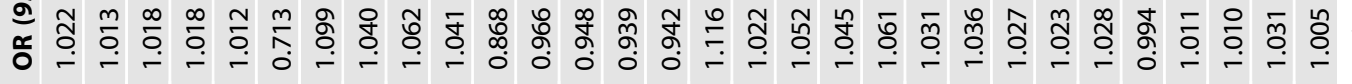

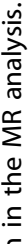

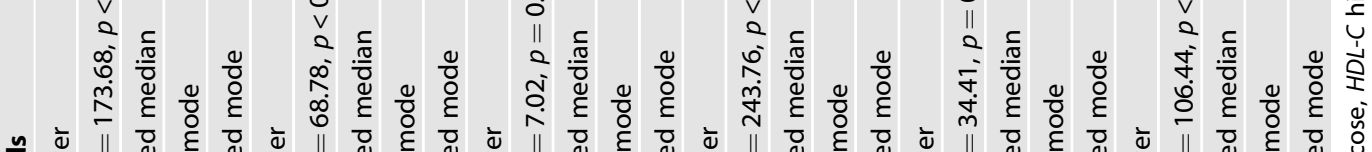

ō

à

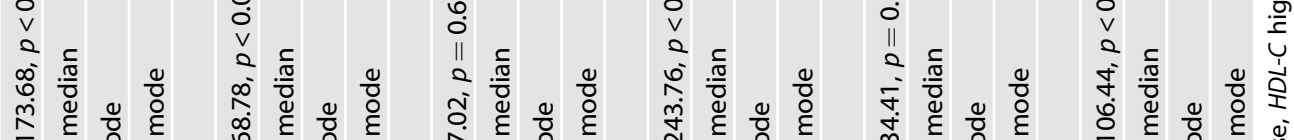

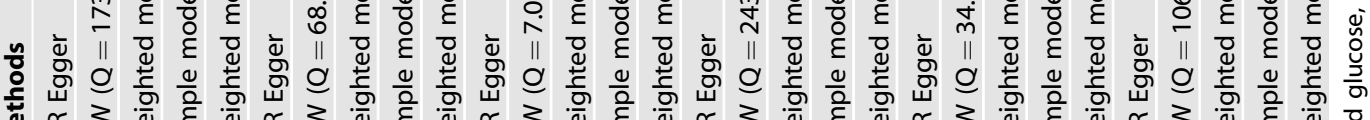

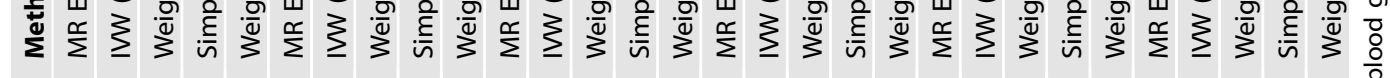

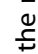

ธ

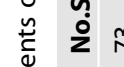

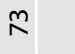

요

으

$\stackrel{\infty}{=}$

๖

8

हे

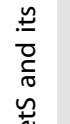

हั

这

$\frac{t}{2}$

흥

产

$\dot{0}$

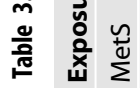
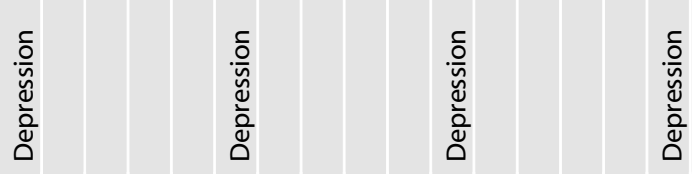

욘
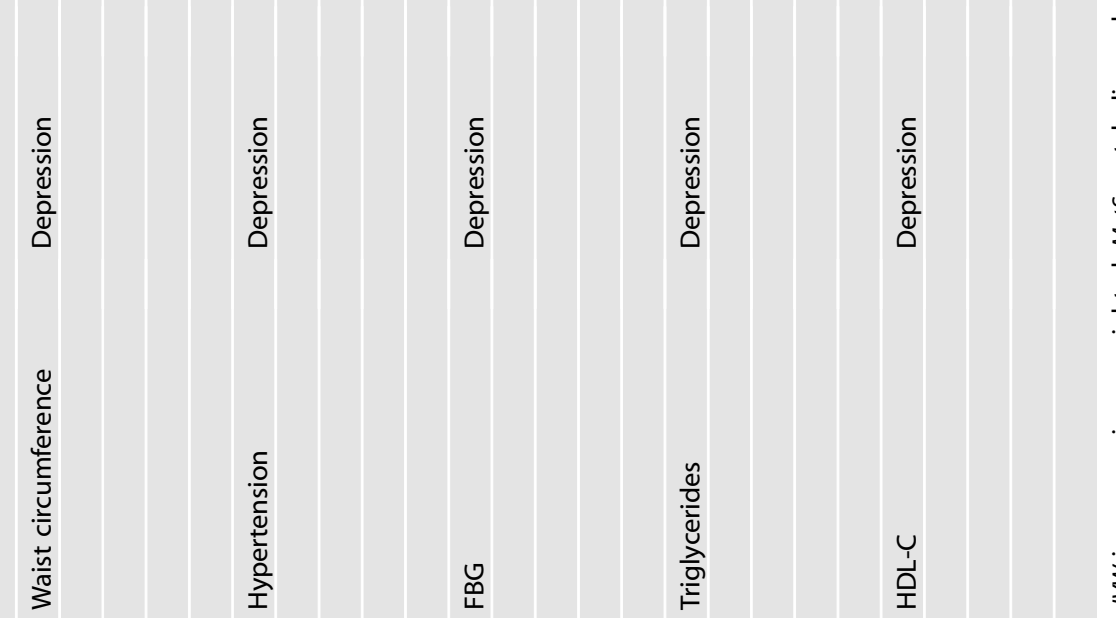
association between them [21, 22, 49]. In contrast, Tang et al. performed an MR analysis using summary-level data from the DIAbetes Genetics Replication And Meta-analysis (DIAGRAM) consortium indicated a positive genetic correlation was present between major depression and type 2 diabetes [62]. This paradox might be due to racial differences, as about $30 \%$ of the population in MAGIC were non-European ancestry. For MetS and its components associated with depression, the evidence from epidemiological studies have been inconsistent [51, 63], and no positive correlations were observed in our MR analyses. However, potential horizontal pleiotropy was founded for the associations between waist circumference and FBG and risk of depression. Therefore, further studies were wanted to investigate the causal associations between these factors.

Although the exact mechanisms underlying depression to MetS remain unclear, different hypotheses have been put forward. Firstly, individuals with depression or major depression are prone to have a sedentary lifestyle and a diet rich in fat or carbohydrates [64], leading to an increased risk of developing MetS. Secondly, depression and MetS share common pathophysiological mechanisms in the stress system, such as autonomic nervous system disorder and abnormal activation of the hypothalamus-pituitary-adrenal (HPA) axis [65]. It has been shown that patients with depression present an increased HPA activity [66], and the dysregulation of the HPA axis could affect MetS via influencing abdominal glucose metabolism, fat accumulation, and blood pressure regulation [23]. For example, the activation of the HPA axis resulted in higher levels of cortisol and cortisone [67]. A typical effect of cortisol is to redistribute adipose tissue around the abdominal area, leading to central obesity in the long term [68]. In addition, findings have supported the critical roles of low-grade systemic inflammation, elevated oxidative and nitrosidative stress in depression and MetS [65]. Thirdly, conventional antidepressants may have direct side effects on MetS and its components [69]. For instance, tricyclic antidepressant (TCA) use increased the risk of MetS and weight gain [70]. The usage of serotonin-norepinephrine reuptake inhibitors (SNRIs) has been associated with a higher risk for hypertension [71].

Traditional observational studies suggested a bidirectional relationship between depression and MetS. It might be biased by potential confounding and reverse causality, such as unhealthy lifestyle, antidepressant usage. A key strength of this MR study is that our findings avoided reverse causality and minimized residual confounding. Another strength is that we implemented the most comprehensive dataset for the exposures and the most extensive summary-level data for risk of depression, MetS, and its components, therefore, the power to investigate causal associations was high and the estimated effect magnitudes were more accurately. However, our study also has limitations. First, the functions of the genetic instruments and how they affect the risk factors were not fully understood. Second, we still can't remove the potential pleiotropy effect that might be concealed by the small number of genetic instruments or small sample size, although the MR-egger intercept showed little horizontal pleiotropism. Third, obvious heterogeneities obtained from individual variants by the Cochran's $Q$ value were present in the MR analyses. We thus performed the leave-one-out analysis, and the results indicated the stability of the observed associations.

In conclusion, our bidirectional MR study indicated a causal link between depression and MetS (and its components), the reverse direction showed no causal associations. Our findings recommended that the prevention, management, and treatment for depression might be enhanced for MetS and its components prevention.

\section{REFERENCES}

1. Bromet E, Andrade LH, Hwang I, Sampson NA, Alonso J, de Girolamo G, et al. Cross-national epidemiology of DSM-IV major depressive episode. BMC Med. 2011;9:90.
2. Lim GY, Tam WW, Lu Y, Ho CS, Zhang MW, Ho RC. Prevalence of depression in the community from 30 countries between 1994 and 2014. Sci Rep. 2018;8:2861.

3. Kessler RC, Bromet EJ. The epidemiology of depression across cultures. Annu Rev Public Health. 2013;34:119-38.

4. Friedrich MJ. Depression is the leading cause of disability around the world. JAMA. 2017;317:1517.

5. Disease GBD, Injury I, Prevalence C. Global, regional, and national incidence, prevalence, and years lived with disability for 310 diseases and injuries, 19902015: a systematic analysis for the Global Burden of Disease Study 2015. Lancet. 2016;388:1545-602.

6. Beck A, Crain AL, Solberg LI, Unutzer J, Glasgow RE, Maciosek MV, et al. Severity of depression and magnitude of productivity loss. Ann Fam Med. 2011;9:305-11.

7. Ribeiro JD, Huang X, Fox KR, Franklin JC. Depression and hopelessness as risk factors for suicide ideation, attempts and death: meta-analysis of longitudinal studies. Br J Psychiatry. 2018;212:279-86.

8. Hawton K, Casanas ICC, Haw C, Saunders K. Risk factors for suicide in individuals with depression: a systematic review. J Affect Disord. 2013;147:17-28.

9. Center C, Davis M, Detre T, Ford DE, Hansbrough W, Hendin $\mathrm{H}$, et al. Confronting depression and suicide in physicians: a consensus statement. JAMA. 2003;289:3161-6.

10. Gilman SE, Sucha E, Kingsbury M, Horton NJ, Murphy JM, Colman I. Depression and mortality in a longitudinal study: 1952-2011. CMAJ 2017;189:E1304-E1310.

11. Mata DA, Ramos MA, Bansal N, Khan R, Guille C, Di Angelantonio E, et al. Prevalence of depression and depressive symptoms among resident physicians: a systematic review and meta-analysis. JAMA 2015;314:2373-83.

12. Eckel RH, Alberti KG, Grundy SM, Zimmet PZ. The metabolic syndrome. Lancet. 2010;375:181-3.

13. Saklayen MG. The global epidemic of the metabolic syndrome. Curr Hypertens Rep. 2018;20:12.

14. Tan CE, Ma S, Wai D, Chew SK, Tai ES. Can we apply the National Cholesterol Education Program Adult Treatment Panel definition of the metabolic syndrome to Asians? Diabetes Care. 2004;27:1182-6.

15. Gonzalez-Chavez A, Chavez-Fernandez JA, Elizondo-Argueta S, Gonzalez-Tapia A, Leon-Pedroza Jl, Ochoa C. Metabolic syndrome and cardiovascular disease: a health challenge. Arch Med Res. 2018;49:516-21.

16. Hildrum B, Mykletun A, Dahl AA, Midthjell K. Metabolic syndrome and risk of mortality in middle-aged versus elderly individuals: the Nord-Trondelag Health Study (HUNT). Diabetologia 2009;52:583-90.

17. Rethorst CD, Leonard D, Barlow CE, Willis BL, Trivedi MH, DeFina LF. Effects of depression, metabolic syndrome, and cardiorespiratory fitness on mortality: results from the Cooper Center Longitudinal Study. Psychol Med. 2017;47:2414-20.

18. Vancampfort D, Vansteelandt K, Correll CU, Mitchell AJ, De Herdt A, Sienaert P, et al. Metabolic syndrome and metabolic abnormalities in bipolar disorder: a metaanalysis of prevalence rates and moderators. Am J Psychiatry. 2013;170:265-74.

19. Vaccarino V, McClure C, Johnson BD, Sheps DS, Bittner V, Rutledge T, et al. Depression, the metabolic syndrome and cardiovascular risk. Psychosom Med. 2008;70:40-8.

20. Dregan A, Rayner L, Davis KAS, Bakolis I, Arias de la Torre J, Das-Munshi J, et al. Associations between depression, arterial stiffness, and metabolic syndrome among adults in the UK Biobank population study: a mediation analysis. JAMA. Psychiatry. 2020;77:598-606.

21. Moreira FP, Jansen $K$, Cardoso TA, Mondin TC, Vieira IS, Magalhaes $P$, et al Metabolic syndrome, depression and anhedonia among young adults. Psychiatry Res. 2019;271:306-10.

22. Moreira FP, Jansen $K$, Cardoso TA, Mondin TC, Magalhaes $P$, Kapczinski $F$, et al. Metabolic syndrome in subjects with bipolar disorder and major depressive disorder in a current depressive episode: population-based study: metabolic syndrome in current depressive episode. J Psychiatr Res. 2017;92:119-23.

23. Pan A, Keum N, Okereke Ol, Sun Q, Kivimaki M, Rubin RR, et al. Bidirectional association between depression and metabolic syndrome: a systematic review and meta-analysis of epidemiological studies. Diabetes Care. 2012;35:1171-80.

24. Ghanei Gheshlagh R, Parizad N, Sayehmiri K. The relationship between depression and metabolic syndrome: systematic review and meta-analysis study. Iran Red Crescent Med J. 2016;18:e26523.

25. Moradi Y, Albatineh AN, Mahmoodi H, Gheshlagh RG. The relationship between depression and risk of metabolic syndrome: a meta-analysis of observational studies. Clin Diabetes Endocrinol. 2021;7:4.

26. Vancampfort $D$, Correll CU, Wampers M, Sienaert $P$, Mitchell AJ, De Herdt A, et al. Metabolic syndrome and metabolic abnormalities in patients with major depressive disorder: a meta-analysis of prevalences and moderating variables. Psychol Med. 2014;44:2017-28.

27. Smith GD, Ebrahim S. 'Mendelian randomization': can genetic epidemiology contribute to understanding environmental determinants of disease? Int J Epidemiol. 2003;32:1-22.

28. Emdin CA, Khera AV, Kathiresan S. Mendelian randomization. JAMA 2017:318:1925-6. 
29. Ziegler A, Mwambi H, Konig IR. Mendelian randomization versus path models: making causal inferences in genetic epidemiology. Hum Hered. 2015;79:194-204.

30. Lawlor DA, Harbord RM, Sterne JA, Timpson N, Davey Smith G. Mendelian randomization: using genes as instruments for making causal inferences in epidemiology. Stat Med. 2008;27:1133-63.

31. Davies NM, Holmes MV, Davey Smith G. Reading mendelian randomisation studies: a guide, glossary, and checklist for clinicians. BMJ. 2018;362:k601.

32. Davey Smith G, Hemani G. Mendelian randomization: genetic anchors for causal inference in epidemiological studies. Hum Mol Genet. 2014;23:R89-98.

33. Zhao Q, Chen Y, Wang J, Small DS. Powerful three-sample genome-wide design and robust statistical inference in summary-data Mendelian randomization. Int J Epidemiol. 2019;48:1478-92.

34. Porcu E, Rueger S, Lepik K, e QC, Consortium B, Santoni FA, et al. Mendelian randomization integrating GWAS and eQTL data reveals genetic determinants of complex and clinical traits. Nat Commun. 2019;10:3300.

35. Heng D, Ma S, Lee JJ, Tai BC, Mak KH, Hughes K, et al. Modification of the NCEP ATP III definitions of the metabolic syndrome for use in Asians identifies individuals at risk of ischemic heart disease. Atherosclerosis. 2006;186:367-73.

36. Davey Smith G, Ebrahim S. What can mendelian randomisation tell us about modifiable behavioural and environmental exposures? BMJ. 2005;330:1076-9.

37. Howard DM, Adams MJ, Clarke TK, Hafferty JD, Gibson J, Shirali M, et al. Genomewide meta-analysis of depression identifies 102 independent variants and highlights the importance of the prefrontal brain regions. Nat Neurosci. 2019;22:343-52.

38. Lind L. Genome-wide association study of the metabolic syndrome in UK Biobank. Metab Syndr Relat Disord. 2019;17:505-11.

39. Elsworth B, Mitchell R, Raistrick C, Paternoster L, Hemani G, Gaunt T. MRCIEU UK Biobank GWAS pipeline version 1. 2017. https://doi.org/10.5523/ BRIS.2FAHPKSONT1ZI26XOSYAMQO8RR

40. Shungin D, Winkler TW, Croteau-Chonka DC, Ferreira T, Locke AE, Magi R, et al. New genetic loci link adipose and insulin biology to body fat distribution. Nature. 2015;518:187-96.

41. International Consortium for Blood Pressure Genome-Wide Association $S$, Ehret GB, Munroe PB, Rice KM, Bochud M, Johnson AD, et al. Genetic variants in novel pathways influence blood pressure and cardiovascular disease risk. Nature 2011;478:103-9.

42. Chen J, Spracklen CN, Marenne G, Varshney A, Corbin LJ, Luan J, et al. The transancestral genomic architecture of glycemic traits. Nat Genet. 2021;53:840-60.

43. Richardson TG, Sanderson E, Palmer TM, Ala-Korpela M, Ference BA, Davey Smith $\mathrm{G}$, et al. Evaluating the relationship between circulating lipoprotein lipids and apolipoproteins with risk of coronary heart disease: a multivariable Mendelian randomisation analysis. PLoS Med. 2020;17:e1003062.

44. Willer CJ, Schmidt EM, Sengupta S, Peloso GM, Gustafsson S, Kanoni S, et al. Discovery and refinement of loci associated with lipid levels. Nat Genet. 2013;45:1274-83.

45. Burgess S, Thompson SG, Collaboration CCG. Avoiding bias from weak instruments in Mendelian randomization studies. Int J Epidemiol. 2011;40:755-64.

46. Bowden J, Spiller W, Del Greco MF, Sheehan N, Thompson J, Minelli C, et al. Improving the visualization, interpretation and analysis of two-sample summary data Mendelian randomization via the radial plot and radial regression. Int J Epidemiol. 2018;47:1264-78.

47. Bae SC, Lee YH. Causal association between rheumatoid arthritis and a decreased risk of Alzheimer's disease: a Mendelian randomization study. Z Rheumatol. 2019;78:359-64.

48. Burgess S. Sample size and power calculations in Mendelian randomization with a single instrumental variable and a binary outcome. Int J Epidemiol. 2014;43:922-9.

49. Kim Y, Kim HY. Association between depression and metabolic syndrome in Korean adults: data from the 2014 and 2016 Korea National Health and Nutrition Examination Survey. Asia Pac J Public Health 2019;31:18-29.

50. van Leijden MJ, Penninx B, Agyemang C, Olff M, Adriaanse MC, Snijder MB. The association of depression and posttraumatic stress disorder with the metabolic syndrome in a multi-ethnic cohort: the HELIUS study. Soc Psychiatry Psychiatr Epidemiol. 2018;53:921-30.

51. Virtanen $M$, Ferrie JE, Akbaraly $T$, Tabak A, Jokela M, Ebmeier KP, et al. Metabolic syndrome and symptom resolution in depression: a 5-year follow-up of older adults. J Clin Psychiatry. 2017;78:e1-e7.

52. Olvera RL, Williamson DE, Fisher-Hoch SP, Vatcheva KP, McCormick JB. Depression, obesity, and metabolic syndrome: prevalence and risks of comorbidity in a population-based representative sample of Mexican Americans. J Clin Psychiatry. 2015;76:e1300-5.

53. Akbaraly TN, Ancelin ML, Jaussent I, Ritchie C, Barberger-Gateau P, Dufouil C, et al. Metabolic syndrome and onset of depressive symptoms in the elderly: findings from the three-city study. Diabetes Care. 2011;34:904-9.

54. Akbaraly TN, Kivimaki M, Brunner EJ, Chandola T, Marmot MG, Singh-Manoux A, et al. Association between metabolic syndrome and depressive symptoms in middle-aged adults: results from the Whitehall II study. Diabetes Care. 2009;32:499-504.
55. Kim SY, Park JH, Lee MY, Oh KS, Shin DW, Shin YC. Physical activity and the prevention of depression: a cohort study. Gen Hosp Psychiatry. 2019;60:90-97.

56. Choi KW, Chen CY, Stein MB, Klimentidis YC, Wang MJ, Koenen KC, et al. Assessment of bidirectional relationships between physical activity and depression among adults: a 2-sample Mendelian randomization study. JAMA Psychiatry. 2019;76:399-408.

57. Matta J, Hoertel N, Kesse-Guyot E, Plesz M, Wiernik E, Carette C, et al. Diet and physical activity in the association between depression and metabolic syndrome: constances study. J Affect Disord. 2019;244:25-32.

58. Vanhala M, Jokelainen J, Keinanen-Kiukaanniemi S, Kumpusalo E, Koponen H. Depressive symptoms predispose females to metabolic syndrome: a 7-year follow-up study. Acta Psychiatr Scand. 2009;119:137-42.

59. Xu Q, Anderson D, Lurie-Beck J. The relationship between abdominal obesity and depression in the general population: a systematic review and meta-analysis. Obes Res Clin Pr. 2011;5:e267-360.

60. East C, Willis BL, Barlow CE, Grannemann BD, FitzGerald SJ, DeFina LF, et al. Depressive symptoms and metabolic syndrome in preventive healthcare: the Cooper Center longitudinal study. Metab Syndr Relat Disord. 2010;8:451-7.

61. Bakhtiari A, Hashemi M, Hosseini SR, Omidvar S, Bijani A, Khairkhah F. The relationship between depression and metabolic syndrome in the elderly population: the cohort aging study. Iran J Psychiatry. 2018;13:230-8.

62. Tang B, Yuan S, Xiong Y, He Q, Larsson SC. Major depressive disorder and cardiometabolic diseases: a bidirectional Mendelian randomisation study. Diabetologia 2020;63:1305-11.

63. Miettola J, Niskanen LK, Viinamaki H, Kumpusalo E. Metabolic syndrome is associated with self-perceived depression. Scand J Prim Health Care. 2008;26:203-10.

64. Goldbacher EM, Bromberger J, Matthews KA. Lifetime history of major depression predicts the development of the metabolic syndrome in middle-aged women. Psychosom Med. 2009;71:266-72.

65. Marazziti D, Rutigliano G, Baroni S, Landi P, Dell'Osso L. Metabolic syndrome and major depression. CNS Spectr. 2014;19:293-304.

66. Hirtz R, Libuda L, Hinney A, Focker M, Buhlmeier J, Antel J, et al. Lack of evidence for a relationship between the hypothalamus-pituitary-adrenal and the hypothalamus-pituitary-thyroid axis in adolescent depression. Front Endocrinol. 2021;12:662243.

67. Stetler C, Miller GE. Depression and hypothalamic-pituitary-adrenal activation: a quantitative summary of four decades of research. Psychosom Med. 2011;73:114-26.

68. Silarova B, Giltay EJ, Van Reedt Dortland A, Van Rossum EF, Hoencamp E, Penninx BW, et al. Metabolic syndrome in patients with bipolar disorder: comparison with major depressive disorder and non-psychiatric controls. J Psychosom Res. 2015;78:391-8.

69. Mazereel V, Detraux J, Vancampfort D, van Winkel R, De Hert M. Impact of psychotropic medication effects on obesity and the metabolic syndrome in people with serious mental illness. Front Endocrinol. 2020;11:573479.

70. van Reedt Dortland AK, Giltay EJ, van Veen T, Zitman FG, Penninx BW. Metabolic syndrome abnormalities are associated with severity of anxiety and depression and with tricyclic antidepressant use. Acta Psychiatr Scand. 2010;122:30-9.

71. Zhong Z, Wang L, Wen X, Liu Y, Fan Y, Liu Z. A meta-analysis of effects of selective serotonin reuptake inhibitors on blood pressure in depression treatment: outcomes from placebo and serotonin and noradrenaline reuptake inhibitor controlled trials. Neuropsychiatr Dis Treat. 2017;13:2781-96.

\section{ACKNOWLEDGEMENTS}

Quality Control filtering of the UK Biobank data was conducted by R.Mitchell, G. Hemani, T.Dudding, and L.Paternoster as described in the published protocol (https:// doi.org/10.5523/bris.3074krb6t2frj29yh2b03×3wxj). We thank the PGC, MAGIC, and MRC-IEU UK Biobank for providing the summary-level data used in this study. This study was supported by the medical research projects sponsored by the Health Bureau of Chongqing Municipality and Chongqing Science and Technology Commission (2020MSXM042) and the technology promotion projects sponsored by the Health Bureau of Chongqing Municipality (2020jstg026). The funding agencies of this study had no role in study design, data collection, data management, data analysis, data interpretation, writing of the manuscript, or the decision for submission.

\section{AUTHOR CONTRIBUTIONS}

L.P. had full access to all the data and was responsible for the integrity and accuracy of the data. M.Z. design the study, interpreted the results, and wrote the first draft of the manuscript. L.W. and Z.Y. collected data and performed the data analysis. J.C. improved the study design and interpreted the results. All of the authors critically revised the manuscript and approved the final manuscript. 


\section{COMPETING INTERESTS}

The authors declare no competing interests.

\section{ADDITIONAL INFORMATION}

Supplementary information The online version contains supplementary material available at https://doi.org/10.1038/s41398-021-01759-z.

Correspondence and requests for materials should be addressed to Lihua Peng.

Reprints and permission information is available at http://www.nature.com/ reprints

Publisher's note Springer Nature remains neutral with regard to jurisdictional claims in published maps and institutional affiliations.
Open Access This article is licensed under a Creative Commons Attribution 4.0 International License, which permits use, sharing, adaptation, distribution and reproduction in any medium or format, as long as you give appropriate credit to the original author(s) and the source, provide a link to the Creative Commons license, and indicate if changes were made. The images or other third party material in this article are included in the article's Creative Commons license, unless indicated otherwise in a credit line to the material. If material is not included in the article's Creative Commons license and your intended use is not permitted by statutory regulation or exceeds the permitted use, you will need to obtain permission directly from the copyright holder. To view a copy of this license, visit http://creativecommons. org/licenses/by/4.0/.

(c) The Author(s) 2021 